\title{
Principios Éticos y Prevención Cuaternaria: ¿es posible no proteger el ejercicio del principio de autonomía?
}

\author{
Princípios Éticos e Prevenção Quaternária: é possível não proteger o exercício do princípio da autonomia? \\ Ethical Principles and Quaternary Prevention: is it possible not to protect the exercise of the principle of autonomy?
}

Enrique Miguel Pizzanelli Báez. Facultad de Medicina de la Universidad de la República (UDELAR). Unidad Docente Asistencial Rural de Florida, Florida, Uruguay. miguelpizzanelli@gmail.com (Autor correspondente)

\section{Resumen}

Este artículo tiene como objetivo discutir los principios éticos y la Prevención Cuaternaria utilizando como ejemplo la política adoptada por el gobierno Uruguayo, que a partir del año 2006 obliga a realizar tamizaje para el cáncer de mama, mediante mamografías obligatorias cada dos años, a las trabajadoras entre los 40 y 59 años de edad. Actualmente existe una controversia internacional sobre la pertinencia de los programas de detección precoz del cáncer de mama, y surgen autores e instituciones advirtiendo sobre la tensión entre el ejercicio de la autonomía individual y un contexto que promueve cada vez más la medicalización de la vida. En este contexto es fundamental que los médicos de familia utilicen un abordaje individualizado que respete la autonomía de las personas.

\section{Resumo}

Este artigo tem como objetivo discutir os princípios éticos e a Prevenção Quaternária utilizando como exemplo a política adotada pelo governo uruguaio que, a partir de 2006, obriga o rastreamento para câncer de mama, por meio de mamografias obrigatórias a cada dois anos, para mulheres trabalhadoras na faixa etária entre 40 e 59 anos. Atualmente há uma controvérsia internacional sobre a pertinência dos programas de detecção precoce para câncer de mama e surgem autores e instituições alertando sobre a tensão entre 0 exercício individual da autonomia em um contexto que promove cada vez mais medicalização da vida. Neste contexto, é fundamental que os médicos de família utilizem uma abordagem individualizada que respeite a autonomia das pessoas.

\section{Abstract}

This article aims to discuss the ethical principles and Quaternary Prevention using a policy adopted by the Uruguayan government as example. This policy, which was created in 2006, instituted mass screening for breast cancer by means of compulsory mammography every two years, for women workers aged 40 to 59 years old. However, currently there is an international controversy over the relevance of breast cancer early detection programs, and some authors and institutions are raising concerns about the exercise of individual autonomy within a context that promotes the medicalization of life. In this context, it is fundamental that family physicians use an individualized approach that respects personal autonomy.

Palabras clave: Tamizaje Masivo Mamografía Medicalización Neoplasias de la Mama Autonomía Personal

\section{Palavras-chave:} Programas de Rastreamento Mamografia Medicalização Neoplasias da Mama Autonomia Pessoal

\section{Keywords:} Mass Screening Mammography Medicalization Breast Neoplasms Personal Autonomy 
Preventive medicine displays all 3 elements of arrogance. First, it is aggressively assertive, pursuing symptomless individuals and telling them what they must do to remain healthy. Occasionally invoking the force of law (immunizations, seat belts), it prescribes and proscribes for both individual patients and the general citizenry of every age and stage. Second, preventive medicine is presumptuous, confident that the interventions it espouses will, on average, do more good than harm to those who accept and adhere to them. Finally, preventive medicine is overbearing, attacking those who question the value of its recommendations. (Sackett, 2002, p. 1). ${ }^{1}$

\section{¿Principio de Autonomía cuestionado?}

Durante el año 2013 bajo el título "Uruguay's mandatory breast cancer screening for working women aged 40-59 is challenged" fue publicado un artículo en el British Medical Journal. ${ }^{2}$ Esta comunicación hace referencia a un caso particular. Una usuaria del sistema de salud ha enviado una nota al Ministerio de Salud Pública, solicitando que no se le realice la mamografía exigida como parte del programa de tamizaje al que son sometidas todas las trabajadoras uruguayas. Desde el mes de abril de 2013 hasta el momento de escribir este documento (noviembre), la autoridad sanitaria nacional no ha emitido ninguna respuesta a la solicitud de la usuaria, que en pleno ejercicio de sus facultades y sabiendo que no dańa la salud colectiva, no desea someterse, en esta ocasión, a la realización del mencionado estudio radiológico.

\section{Normas vigentes}

En Uruguay, de acuerdo a lo dispuesto por el decreto presidencial número 571/006 del 19 de diciembre de 2006, se establece la obligatoriedad de realizar el carné de salud. Las actividades incluidas, en dicho carné, son: el monitoreo del estado de salud a través de una entrevista médica, cuestionario que permite identificar factores de riesgo y patologías presentes, así como la realización de pruebas y exámenes de tamizaje o screening para cáncer de mama y cáncer de cuello de útero. Esto implica la realización de mamografía y test citológico de Papanicolaou (PAP) a todas las mujeres trabajadoras, según normas nacionales (establecidas por el Programa Nacional de Control del Cáncer - PRONACCAN), de acuerdo con su edad. . $^{3,4}$

El decreto hace referencia, a su vez, a una ordenanza del Ministerio de Salud Pública, no 402, que establece que estos exámenes se realicen, sin costo ańadido, a las usuarias beneficiarias de prestadores del subsector privado. A su vez, se definen los rangos de edad en los que dichos estudios deben realizarse. En el caso del PAP será aplicado a mujeres no histerectomizadas entre los 21 y 65 años. Respecto a las mamografías, cito textualmente: "Dispónese que el estudio de Mamografía, sin costo de prepago por el Sub-Sistema Privado, de acuerdo a las recomendaciones internacionales, se realizará a las mujeres entre cuarenta y cincuenta y nueve años, cada dos años cuando los estudios son normales".

De este modo, el empleador debe reclamar a cada una de sus empleadas para que cumpla con el procedimiento que implica la realización del llamado "carné de salud". En caso de no cumplirse la normativa, se aplican sanciones tanto para la empresa como para las trabajadoras. Por ejemplo, el empleador puede cesar el pago del salario hasta que se cumpla con el requisito.

\section{Controversia internacional respecto a la pertinencia de los programas de 'detección precoz' para cáncer de mama por medio de mamografía}

Desde hace varios ańos existe controversia internacional sobre la pertinencia de los programas de detección precoz para cáncer de mama. El aporte de evidencias en contra de la eficiencia del cribado por mamografía ha sido liderado por el Centro Cochrane Nórdico. La primera comunicación científica surgida desde este centro, que levantó cierta polvareda, fue publicada en el 2000. Analizó, mediante una revisión sistemática, varios estudios aleatorizados que incluían medio millón de mujeres de varios países y procedencias (Estados Unidos, Escocia, Canadá y Suecia). Los autores se enfrentaron con bastantes dificultades a la hora de comunicar dichos resultados, que no fueron "bienvenidos" (una suerte de literatura gris). Incluso los mismos editores de Cochrane pusieron en peligro la libertad académica de los investigadores. ${ }^{6}$ En el resumen del trabajo realizado por Gøtzsche y Olsen publicado en The Lancet ${ }^{7}$ se señala: 
Screening for breast cancer with mammography is unjustified. If the Swedish trials are judged to be unbiased the data show that for every 1000 women screened biennially throughout 12 years, one breast-cancer death is avoided whereas the total number of deaths is increased by six. If the Swedish trials (apart from the Malmo trial) are judged to be biased, there is no reliable evidence that screening decreases breast-cancer mortality. (Gøtzsche P, Olsen O, 2000, p. 1). ${ }^{7}$

"El screening para cáncer de mama con mamografía es injustificado": con esta contundente sentencia inician su apartado de interpretaciones. Después de esta publicación, la cascada de trabajos centrados en el análisis sobre la pertinencia de la detección precoz del cáncer de mama por mamografía, surgidos desde el Centro Nórdico de Cochrane, ha sido abrumadora tanto en cantidad como en calidad. ${ }^{8,9}$ El metanálisis del año 2000 encuentra, en cuatro de un total de ocho estudios, sesgos e inconsistencias en el proceso de aleatorización. Estos estudios analizados fueron los documentos principales que, en su momento, condujeron a justificar la práctica del screening. En los dos ensayos donde la aleatorización fue realizada adecuadamente, no se encontraron efectos del cribado en la reducción de la mortalidad por cáncer de mama.

\section{Es importante reflexionar sobre las tensiones a las que nos expone la medicalización de la vida}

La discusión de la tensión entre el ejercicio individual de la autonomía y el bien común es el centro del debate. La medicalización de la vida tiene múltiples protagonistas: los profesionales; las instituciones; las empresas médico-farmacéuticas y de tecnologías médicas; los medios masivos de comunicación, la población usuaria del sistema de salud; los organismos que ejercen rectoría y los administradores de servicios sanitarios. Estos protagonismos promueven acciones con movimientos que oscilan entre diferentes valores e intereses. ${ }^{10}$

Quisiera concentrarme en factores de orden cultural que intervienen en la medicalización. La reforma de salud en Uruguay, que ha surgido desde el Estado, promueve un cambio en el modelo asistencial. Como todo modelo, está sustentado en un paradigma de salud. Por lo tanto, hay un movimiento o evolución desde un paradigma de salud imperante a otro nuevo, con valores que se pretenden impulsar. Con la ley de reforma de salud del ańo $2008^{11}$ se ha impulsado mayor equidad y acceso a la salud, con destacables logros. Ha sido un camino de mejora y ha impulsado ciertos valores en el sistema que estábamos esperando y deseando. Sin embargo, las decisiones en los procesos de cambio recorren diversos caminos. Dichas decisiones son tomadas, la mayor parte de las veces, por sanitaristas y políticos que creen que es mejor proponer algo que no hacer nada, y no tienen en cuenta la evidencia científica que va en contra de lo que ellos consideran una "buena política". La mayoría de los usuarios avalan estas decisiones porque el nuevo paradigma es una construcción colectiva y es, desde ahí, desde donde se analizan los fenómenos. Las otras miradas o alternativas que emerjan serán resistidas y podrán ser interpretadas como "contraculturales", tales como, el planteamiento de gozar del ejercicio de la autonomía y, por tanto, de negarse a cumplir una medida impuesta.

\section{No siempre más es mejor: Ley de los Cuidados Inversos}

La mortalidad por cáncer de mama a nivel mundial es del 9\%, y Uruguay casi triplica este número. Cuando se valoran tales datos estadísticos, los responsables por tomar decisiones pueden sensibilizarse y promover acciones de alcance colectivo con pretensión preventiva, e incluso de carácter obligatorio.

La creciente propuesta preventiva satura la agenda de los equipos de salud del primer nivel de atención y provoca un agotamiento de recursos por extrema competencia. Se desvía buena parte de la energía desde lo que es realmente prioritario hacia lo que es sistemáticamente impuesto. Podemos caer en la contradicción de que a pesar de querer dar más, estemos privando de mucho a los que más necesitan de nuestra atención: los vulnerables y excluidos. Podemos llegar a un desequilibrio de recursos que aumente la inequidad, tal y como plantea Tudor Hart en su Ley de los Cuidados Inversos: "la disponibilidad de los recursos varía inversamente a las necesidades". ${ }^{2}$ 


\section{Atención centrada en la enfermedad versus atención centrada en la persona}

Las acciones desencadenadas por los programas preventivos y de screening responden muy bien a una modalidad de abordaje asistencial centrado en la enfermedad. Es una perspectiva pragmática posible, pero no deja de ser una perspectiva reduccionista, ya que compromete las acciones de los proveedores de salud, limitándolas a una búsqueda de enfermedades en sujetos sanos, mediante la aplicación de protocolos, cuestionarios y el uso de tecnología. Y todo esto, para encontrar aquello "de lo que no se sufre". Las actividades sanitarias se restringen a una función medicalizadora, que puede comprometer el enfoque integral centrado en la persona. El carácter obligatorio de alguna de estas medidas no respeta la intimidad ni el principio de autodeterminación de los individuos. El cuerpo del ser humano no es una propiedad colectiva.

\section{Como proteger el Principio de Autonomía}

Somos médicos familiares y comunitarios con responsabilidad en el cuidado y mantenimiento de la salud de las personas, y como tales, nuestros esfuerzos se focalizan en varios ejes fundamentales: integralidad, atención longitudinal, compromiso con la persona, respeto por la alteridad. Trabajar con esta orientación no impide el abordaje de problemas y enfermedades, sino que lo integra desde una dialéctica contextualizadora. Este modo de abordaje estratégico de la salud es el que realizamos a diario en la actividad clínica comunitaria, pues nos permite identificar factores de riesgo particulares y definir priorizaciones individualizadas.

Requiere establecer una agenda común paciente-médico, compartir las miradas sobre el problema y establecer prioridades para las acciones o planes a acordar. Este enfoque evita la fragmentación asistencial, permite detectar personas en riesgo de medicalización ${ }^{13,14} \mathrm{y}$ admite trabajar desde una perspectiva de reducción de daños. ${ }^{15}$

\section{Abordaje Estratégico Centrado en la Persona}

- Implica una mirada multidimensional sobre la persona para lograr una interacción y comunicación eficaz y asertiva.

- Incluye al vínculo como herramienta y a los aspectos relacionales que implican compromiso, empatía y ajustes permanentes de la distancia óptima en la relación paciente-médico-familia.

- Implica una actitud de respeto hacia la autonomía y el espacio personal del otro.

- Tiene en cuenta la perspectiva y la opinión del paciente, tanto en los aspectos terapéuticos como en los preventivos, compartiendo, de este modo, la toma de decisiones.

- Permite priorizar los problemas o situaciones en las que es necesario intervenir.

El abordaje estratégico centrado en la persona se configura, entonces, como proceso protector ante esta creciente escalada de "arrogancia preventiva", que es sostenida por posicionamientos autoritarios y paternalistas, totalmente funcionales para una medicina utilitaria pero que termina erosionando la autonomía de los pacientes.

La comunicación a través de redes entre colegas de varios países nos ha permitido, en estos últimos meses, exponer e intercambiar nuestras inquietudes, discrepancias y reflexiones, incluso exponiéndolas en actividades científicas, con la finalidad de promover un pensamiento crítico y reflexión entre pares. ${ }^{16,17}$

Un enfoque respetuoso hacia la individualidad y la autonomía, que brote con naturalidad desde el vínculo del paciente con su terapeuta, que tome como centro la person ${ }^{18}$ y no la enfermedad, tal vez sea el mejor factor protector de la salud con el que podamos contribuir desde nuestra práctica. 


\section{Referencias}

1. Sacket D. Hormone replacement therapy: the arrogance of preventive medicine. CMAJ. 2002;67(4):363-364. Disponible en: http://www.cmaj.ca/content/167/4/363.full.pdf.

2. Arie S. Uruguay's mandatory breast cancer screening for working women aged 40-59 is challenged. BMJ. 2013;346:f1907. http://dx.doi.org/10.1136/bmj.f1907

3. Ministerio de Salud Pública (UY); Archivo Presidencia de la República Oriental. Decreto n 571/006. Montevideo: Ministerio de Salud Pública; 2013 [última consulta el 2013 Nov 10]. Disponible en: http://archivo.presidencia.gub.uy/_Web/decretos/2006/12/S253_15_09_2006_00001.PDF.

4. Ministerio de Salud Pública (UY). Decreto n 571/006: carné de salud básico a las mujeres. Expedición. Montevideo: El Derecho Digital; 2013 [última consulta el 2013 Nov 10]. Disponible en: http://www.elderechodigital.com.uy/smu/legisla/D0600571.html.

5. Ministerio de Salud Pública (UY). Ordenanza n 402, 11 de julio de 2006. Montevideo: Ministerio de Salud Pública; 2006 [última consulta el 2013 Nov 10]. Disponible en: http://www2.msp.gub.uy/andocasociado.aspx?5684,24733

6. Horton R. Screening mammography: an overview revisited. Lancet. 2001;358(9290):1284-1285. Disponible en: http://www.miniseminaires.com/wp-content/uploads/2009/05/4-screening-mammography-is-justifiable.pdf http://dx.doi.org/10.1016/S0140-6736(01)06452-2

7. Gøtzsche P, Olsen O. Is screening for breast cancer with mammography justifiable? Lancet. 2000;355(9198):129-134. http://dx.doi.org/10.1016/S0140-6736(99)06065-1

8. Zahl P, Gøtzsche P, Mæhlen J. Natural history of breast cancers detected in the Swedish mammography screening programme: a cohort study. Lancet Oncol. 2011;12(12):1118-1124. http://dx.doi.org/10.1016/S1470-2045(11)70250-9

9. Gøtzsche P, Jørgensen J. Screening for breast cancer with mammography. Cochrane Database Syst Rev. 2013;(6):CD001877. http://dx.doi.org/10.1002/14651858.CD001877.pub5

10. Márquez S, Meneu R. La medicalización de la vida y sus protagonistas. GCS. 2003;5(2):47-53 Disponible en: http://www.iiss.es/gcs/gestion16.pdf.

11. Sistema Nacional Integrado de Salud (UY). Ley n 18.211. Normativa referente a su creación, funcionamiento y financiación. Montevideo: Sistema Nacional Integrado de Salud; 2007. Disponible en: http://www.parlamento.gub.uy/leyes/AccesoTextoLey.asp?Ley=18211\&Anchor=.

12. Hart JT. The inverse care law. Lancet. 1971;1:405-412. Disponible en: http://www.juliantudorhart.org/papers/Paper11.pdf.

13. Kuehlein T, Sghedoni D, Visentin G, Gervas J, Jamoulle M. Prevención cuaternaria, actividad del médico general. Prim Care. 2010;10(18):350-354. Disponible en: http://www.primary-care.ch/docs/primarycare/archiv/de/2010/2010-18/2010-18-368_ELPS_esp.pdf.

14. Nève J, Bernstein J, Terra MA. Prevención cuaternaria, una tarea explícita del médico generalista: una entrevista con Marc Jamoulle. AMFG. 2013;10(2):23-26. Disponible en: http://archivos.famfyg.org/revista/index.php/amfyg/article/view/130.

15. Jamoulle M, Gomes L. Prevenção quaternária e limites em medicina. In: $18^{\circ}$ Congresso Nacional de MGF, $12^{\circ}$ Encontro Nacional de Internos de MGF e Jovens MF; 2013, Covilha.

16. Pizzanelli M. Estan cambiando los tiempos. Uruguay; 2013 [última consulta el 2013 Nov 10]. Disponible en: http://estancambiandolostiempos.blogspot.com.br/2013/02/cribado-cancer-de-mama-un-poco-mas-de.html.

17. Pizzanelli M. O rastreamento do câncer de mama e a política adotada pelo Uruguai. In: $12^{\circ}$ Congresso Brasileiro de Medicina de Família e Comunidade; 2013, Belém do Pará. Disponible en: http://www.slideshare.net/MiguelPizzanelli/cribado-cancer-mamabelem2013.

18. Lopes JMC. Consulta e abordagem centrada na pessoa. In: Gusso GDF, Lopes JMC. Tratado de medicina de família e comunidade: princípios, formação e prática. Porto Alegre: Artmed; 2012. p. 113-123. 